\title{
Deep Vein Thrombosis in Non-ICU COVID-19 Patients: An Additional Risk
}

\author{
Sohil Pothiawala ${ }^{1, *}$
}

\author{
${ }^{1}$ Department of Emergency Medicine, \\ Woodlands Health Campus, Singapore \\ *Correspondence \\ drsohilpothiawala@yahoo.com \\ (Sohil Pothiawala)
}

\begin{abstract}
Introduction: Venous thromboembolism (VTE) encompasses two conditions, deep vein thrombosis (DVT) and pulmonary embolism (PE). Patients admitted to the intensive care unit (ICU) are at highest thrombotic risk. The incidence of VTE in COVID-19 patients is not definitely established, with studies reporting an incidence rate of $25-27 \%$. Discussion: As the COVID-19 pandemic progresses, studies are reporting increased incidence of VTE in COVID-19 patients admitted in ICU. But there are not many studies reporting the incidence of DVT in non-ICU COVID-19 patients. Elevated levels of D-dimer indicate hypercoagulability, but it can also indicate inflammation. $68 \%$ of hospitalized COVID-19 patients had elevated D-dimer levels. Thus, D-dimer level at the time of admission does not co-relate with the development of VTE or its related complications in COVID-19 patients. One study reported that out of the total number of non-ICU COVID-19 patients who had signs or symptoms suggestive of DVT, $50 \%$ were detected to be positive on compression ultrasound scan. While some studies and international guidelines recommend the administration of LMWH in all hospitalized COVID-19 patients, some studies recommend that the physicians should closely monitor patients for development of signs and symptoms of DVT, and perform prompt diagnostic tests to aid early diagnosis and initiate anticoagulation. Hence, the treatment protocols vary widely across institutions, and the decision of thromboprophylaxis is also made on a case-bycase basis. Conclusion: Further studies are needed to establish the incidence of DVT in non-ICU patients, the predictive value of d-dimer levels to detect DVT risk, and an optimal therapeutic regimen of prophylactic anti-coagulant therapy in these patients.
\end{abstract}

\section{Keywords}

COVID-19, Deep vein thrombosis, Non-ICU, Incidence

\section{Introduction}

Venous thromboembolism (VTE) encompasses two conditions, deep vein thrombosis (DVT) and pulmonary embolism (PE). It is a well-known complication of bed-bound or minimally ambulant hospitalized patients. Patients admitted to the intensive care unit (ICU) are at highest thrombotic risk, with the incidence ranging from $15-32 \%[1,2]$. Patients with risk factors like old age, obesity, bedbound in ICU, active malignancy, major surgery, past history of VTE and those with indwelling catheters have a higher risk of developing VTE.

\section{Discussion}

The incidence of VTE in COVID-19 patients is not definitely established, but an increasing number of cases are being reported. Recently published studies have reported an incidence rate of $25-27 \%$ [3, 4]. This is comparable to the VTE incidence in other patients with Disseminated Intravascular Coagulation (DIC) [1].

Till date, the pathogenesis of DVT in COVID-19 pa- 


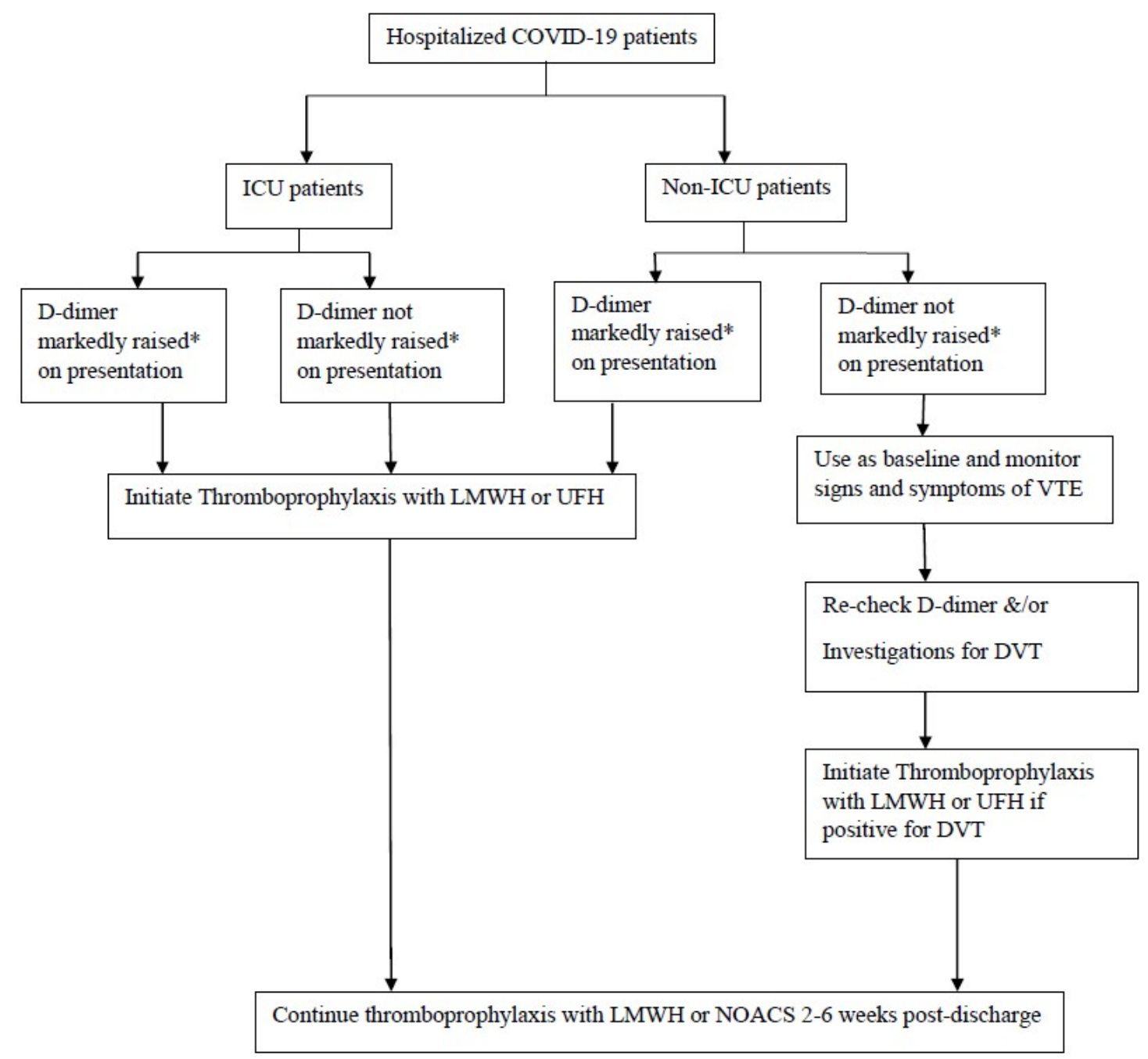

F I G U R E 1. Algorithm for the management of coagulopathy in non-ICU COVID-19 patients.

*A specific cut-off level of D-dimer is not defined, but a three-to-four-fold increase may be considered significant.

tients is not clearly established. Elevated levels of proinflammatory cytokines are found in COVID-19 patients, resulting in cytokine storm. This inflammation causes subsequent activation of coagulation cascade, leading to stimulation of pro-coagulant factors such as D-dimer. This may cause the D-dimer level to be elevated in these patients. The cytokine storm can also potentially cause increased clotting factor production from the liver. The binding of SARS-Cov-2 virus to the angiotensin-converting enzyme2 (ACE-2) receptor on the endothelial cell surface, leads to cellular infiltration and endothelial damage. Together, the resultant effect is increased incidence of thrombosis in COVID-19 patients.

D-dimer level is a well-known, widely used, but nonspecific biomarker for diagnosing VTE. Elevated levels indicate hypercoagulability, but can also indicate inflammation. $68 \%$ of the hospitalized COVID-19 patients had elevated D-dimer levels [5]. Elevated D-dimer level at the time of admission, or a subsequent rise in D-dimer level, are both associated with increased mortality due to COVID-19 $[6,7]$. But, D-dimer levels at presentation do not co-relate with the development of VTE or its related complications [8].

Since the onset of this pandemic, as more information becomes available, increasing number of studies and case reports are being published regarding incidence of pulmonary embolism in COVID-19 ICU patients. One study reported that $23 \%$ patients with severe clinical features of COVID-19 had acute pulmonary embolism on pulmonary CT angiography [9].

But there are limited studies reporting the incidence of DVT in non-ICU COVID-19 patients. One study reported that out of the total number of non-ICU COVID-19 patients who had signs or symptoms suggestive of DVT, $50 \%$ were detected to be positive on compression ultrasound scan. The thrombus was most frequently detected in the iliacfemoral-popliteal veins, followed by the brachial-axillary veins and calf veins [10].

Current guidelines recommend the use of pharmacologic thromboprophylaxis with low-molecular-weight heparin (LMWH) or Unfractionated Heparin (UFH) for ICU patients with confirmed VTE, or those who are acutely 
ill with a high risk of developing VTE [11]. In patients with a history of heparin-induced thrombocytopenia, use of fondaparinux is recommended. In patients with contraindication to the use of anticoagulants, mechanical thromboprophylaxis using pneumatic compression devices must be adopted.

A study by Klok et al [4] recommends pharmacological thrombosis prophylaxis in all COVID-19 patients admitted to the ICU, as they are at high risk of venous thromboembolism [4]. But, the use of thromboprophylaxis in patients who do not have a confirmed diagnosis of thromboembolism is not supported by definite evidence. Some studies recommend administration of prophylactic dose of LMWH in all hospitalized COVID-19 patients in the absence of active bleeding or platelet count $<25 \times 10^{9} / \mathrm{L}$ [7, 12]. There is no clear data suggesting improved outcomes in patients treated with therapeutic anticoagulation. Hence, the treatment protocols vary widely across institutions, and the decision of thromboprophylaxis is also made on a caseby-case basis [13].

Thus, for non-ICU patients, rather than treating all COVID-19 patients with prophylactic anticoagulation, the patients should maintain adequate hydration and they should also be encouraged to mobilize and perform static exercises. Physicians should regularly monitor patients for development of signs and symptoms of DVT, and perform prompt diagnostic tests to aid early diagnosis, initiate anticoagulation and avoid complications (Fig. 1). But due to patient surge during this pandemic, the increasing number of acutely ill patients may be admitted in non-ICU setting to general wards. As these patients would be at higher risk of developing VTE as compared to the low risk patients, they may need to be initiated on prophylactic thromboprophylaxis, even in the absence of confirmed VTE at the time of admission. But this approach needs further evaluation by conducting prospective trials in the non-ICU group of COVID-19 patients, before adopting this anticoagulation approach.

\section{Conclusion}

DVT should be considered a relatively frequent and potentially lethal complication of COVID-19. Further studies are needed to establish its incidence in non-ICU patients, predictive value of d-dimer levels to detect the DVT risk, and an optimal therapeutic regimen of prophylactic anticoagulant therapy in these patients.

\section{ACKNOWLEDGEMENTS}

Thank numerous individuals participated in this study.

\section{CONFLICT OF INTEREST}

The authors declare that there is no conflict of interest regarding the publication of this article.

\section{AUTHOR CONTRIBUTIONS}

SP conceived the idea for the manuscript and also contributed to the writing and editing of the review.

\section{FUNDING INFORMATION}

This research did not receive any specific grant from funding agencies in the public, commercial, or not-for-profit sectors.

\section{REFERENCES}

[1] Levi M, Scully M. How I treat disseminated intravascular coagulation. Blood. 2018;131:845-854.

[2] Cook DJ, Crowther MA, Meade MO, et al. VTE in the ICU workshop participants. Prevalence, incidence, and risk factors for venous thromboembolism in medical-surgical intensive care unit patients. $\mathrm{J}$ Crit Care. 2005;20:309-13.

[3] Cui S, Chen S, Li X, et al. Prevalence of venous thromboembolism in patients with severe novel coronavirus pneumonia. J Thromb Haemost. 2020;18:1421-1424.

[4] Klok FA, Kruip MJHA, Van der Meer NJM, et al. Incidence of thrombotic complications in critically ill ICU patients with COVID19. Thromb Res. 2020;191:145-147.

[5] Zhou F, Yu T, Du R, et al. Clinical course and risk factors for mortality of adult inpatients with COVID-19 in Wuhan, China: a retrospective cohort study. The Lancet. 2020;395:1054-1062.

[6] Connors JM, Levy JH. COVID-19 and its implications for thrombosis and anticoagulation. Blood. 2020;135:2033-2040.

[7] Thachil J, Tang N, Gando S, et al. International Society on Thrombosis and Haemostasis (ISTH) interim guidance on recognition and management of coagulopathy in COVID-19. J Thromb Haemost. 2020;18:1023-1026.

[8] Criel M, Falter M, Jaeken J, et al. Venous thromboembolism in SARSCoV-2 patients: only a problem in ventilated ICU patients, or is there more to it? Eur Respir J. 2020.

[9] Grillet F, Behr J, Calame P, et al. Acute Pulmonary Embolism Associated with COVID-19 Pneumonia Detected by Pulmonary CT Angiography. Radiology. 2020.

[10] Marone E., Rinaldi L. Upsurge of deep venous thrombosis in patients affected by COVID-19: Preliminary data and possible explanations. J Vasc Surg Venous Lymphat Disord. 2020.

[11] Spyropoulos AC, Levy JH, Ageno W, et al. Scientific and Standardization Committee Communication: Clinical Guidance on the Diagnosis, Prevention and Treatment of Venous Thromboembolism in Hospitalized Patients with COVID-19. J Thromb Haemostat. 2020.

[12] American Society of Hematology. COVID-19 and Coagulopathy: Frequently Asked Questions. accessable on: https://www. hematology .org/covid-19/covid-19-and-coagulopathy .

[13] Porfidia A, Pola R. Venous thromboembolism and heparin use in COVID-19 patients: juggling between pragmatic choices, suggestions of medical societies. J Thromb Thrombolysis. 2020.

How to cite this article: Sohil Pothiawala. Deep Vein Thrombosis in Non-ICU COVID-19 Patients: An Additional Risk. Signa Vitae. 2020;16(1):179-181. doi:10.22514/sv.2020.16.0024. 